\title{
Tygerberg Research Ubuntu-Inspired Community Engagement Model: Integrating Community Engagement into Genomic Biobanking
}

\author{
Keymanthri Moodley and Chad Beyer
}

\begin{abstract}
Introduction: Community engagement (CE) is an ethical imperative in research, but the knowledge base for what constitutes effective and ethically sound CE is limited. Ubuntu, as a component of responsive communitarianism where communal welfare is valued together with individual autonomy, is useful in furthering our understanding of effective CE and how it could best be achieved. Similarly, a relative solidarity model serves as a compromise between extreme individualism and extreme communalism and is more appropriate in a heterogenous African context. Approaching CE from an Ubuntu philosophical perspective in southern Africa is particularly important in genomic biobanking, given the implications for individuals, families, and communities.

Discussion: CE is often implemented in a tokenistic manner as an ancillary component of research. Understanding consent information is challenging where genomic biobanking is concerned due to scientific complexity. We started a process of CE around genomic biobanking and conducted empirical research in an attempt to develop a model to promote effective and ethically sound CE, using relative solidarity to create a nuanced application of Ubuntu. The TRUCE model is an eight-step model that uses social mapping to identify potential communities, establishes the scope of $\mathrm{CE}$, and requires that communities are approached early. Co-creation strategies for CE are encouraged and co-ownership of knowledge production is emphasized. Recruiting and engaging communities at each stage of research is necessary. Evaluation and adaptation of CE strategies are included. Discussion and dissemination of results after the research is completed are encouraged.

Conclusions: There is a significant gap between the theory of CE and its authentic application to research in Africa. This Ubuntu-inspired model facilitates bridging that gap and is particularly suited to genomic biobanking. The CE model enhances and complements the consent process and should be integrated into research as a funding and regulatory requirement where applicable.
\end{abstract}

Keywords: biobank, community engagement, genomic, consent

\section{Introduction}

$\mathrm{C}$ OMMUNITY ENGAGEMENT (CE), as a component of Stakeholder Engagement (SE), in biomedical research is increasingly being recognized by researchers and required by some funders as a primary ethical responsibility, with mutually beneficial outcomes for the community and the research team. ${ }^{1-5}$ This importance is emphasized globally by projects such as INVOLVE in the United Kingdom and by PCORI (Patient-Centered Outcomes Research Initiative) in the United States. ${ }^{6-8}$ While generally accepted on principle, the knowledge base for what constitutes effective and ethically sound $\mathrm{CE}$ is limited. ${ }^{9-11}$ Furthermore, there is a concern that $\mathrm{CE}$ in health research is rarely community directed, often tokenistic in practice, and may not amount to genuine engagement, empowerment, or consideration for the diverse, heterogeneous concerns and priorities of these communities. ${ }^{1,5,12-18}$

Department of Medicine, Faculty of Medicine and Health Sciences, Centre for Medical Ethics \& Law, Stellenbosch University, Cape Town, South Africa.

(C) Keymanthri Moodley and Chad Beyer, 2019; Published by Mary Ann Liebert, Inc. This Open Access article is distributed under the terms of the Creative Commons License (http://creativecommons.org/licenses/by/4.0), which permits unrestricted use, distribution, and reproduction in any medium, provided the original work is properly cited. 
The spectrum of engagement can vary from community acknowledgment at a superficial level to community-based action research (CBAR). The focus of this article is on CE, which is a more flexible approach that is not so prescriptive in its intention and outcome. In CBAR, the results of the research are implemented to change the situation being studied, ${ }^{19,20}$ which is beyond the scope of this article.

While CE is acknowledged as being necessary, it is important to start as early as possible and to be nonprescriptive, while ensuring constant evaluation and adaptation of the process, promoting equity in public voices and empowerment of the communities represented. ${ }^{12,21}$ Appropriate CE is likely to build relationships, increase trust, enhance consent processes, and augment the capacity of communities, as occurs in developed country settings. The social contract implemented by UK biobank and the Tissue Trust proposed by Emerson et al. are examples. ${ }^{22,23}$ On the other hand, suboptimal $\mathrm{CE}$ is potentially exploitative and disrespectful to communities. ${ }^{14,24}$

Sadly, the latter scenario is often the case in resourcepoor settings. Consequently, the principles of $\mathrm{CE}$ need to be explicit and include notions such as transparency and respect for the community with genuine reflection on developing authentic research approaches that embrace reciprocity, consensus, and collective decision- making. ${ }^{25} \mathrm{CE}$ principles need to be approached and monitored with a strong degree of public oversight to be effective. ${ }^{26}$

In a southern African context, transparent and practical $\mathrm{CE}$ approaches can be enhanced by taking into account the concept of Ubuntu. ${ }^{27,28}$ This is particularly important in genetics and genomics research where families and communities are central to the science. Just as the ancient Greeks believed that, a society grows great when old men plant trees whose shade they know they shall never sit in, so the notion of Ubuntu, central to many traditional southern African communities, encapsulates a sense of communitarianism. Umuntu ngumuntu ngabantu, a person is a person through other persons, often interpreted as I am because you are, speaks to the African philosophical approach of interconnectedness of humans in society.

While Ubuntu is described as being of southern African origin, ${ }^{29}$ others argue that the notion is widely prevalent in other parts of Africa, but is referred to using different terminology. ${ }^{30-32}$ "'Analytically speaking, 'Ubuntu' is a term used to describe the quality or essence of being a person among many sub-Saharan tribes of the Bantu language family." 33 Communitarianism further affirms that individuals are socially embedded, and that personhood is woven into the community with communal good taking priority over individual priorities. ${ }^{34}$ Eze, however, argues that "identity or subjectivity of the individual and the community is mutually constitutive and hence none is supreme.",33 The extent to which this approach is practiced varies from urban to rural communities and among younger and older individuals in different parts of the continent.

The concept of relative solidarity described by Ogunrin et al. reflects this nuanced application of the principles of Ubuntu. $^{32}$ There has been a generational shift demonstrated between the older African generation who subscribe more commonly to the above communal approach to ethics and research in general, while the youth appear to be more enmeshed with Western Philosophical thought that places a higher value on personal autonomy and selfinterest. $^{31,32}$ The relative solidarity model allows for the amalgamation of these contrasting worldviews. This model works in the context of responsive communitarianism, as opposed to classical or authoritarian communitarianism. The main thesis of responsive communitarianism is that people face a conflict of two major sources of normativity: that of the common good and that of autonomy and rights with neither principle taking preference. ${ }^{30}$

While Ubuntu intersects closely with $\mathrm{CE}$ in southern Africa, the value of reciprocal relationships between researchers and communities has also been emphasized in New Zealand, where drawing on indigenous knowledge and Maori protocols and practices has proven invaluable to those involved in biobank research. This bridging of the communication divide has allowed for a greater practice of coproduction and reciprocity of knowledge. ${ }^{35,36}$

While CE grounded in relative solidarity is important in all types of research in African settings, it has particular relevance in the field of genomic biobanking because these fields of research have medical implications for individuals, families, and communities. Genetic research involves the study of DNA, the basic unit of heredity, while genomic research involves the study of the entire human genetic code, known as the genome. This includes the analysis of human material and requires the recruitment of individuals. ${ }^{37}$

Biobanking involves the collection and storage of biological material, often in the form of blood, saliva, and urine, as well as demographic and health-related donor information. Biospecimens and data are anonymized and stored for future use. The success of genomic biobanking lies in the generation of big data and sharing of biospecimens and data in a global research community. This is well demonstrated in the success of the UK Biobank, which has a cohort of over 500,000 samples that are being successfully used to study the epigenetics and predictive lifestyle factors of cardiovascular health events, in addition to a variety of other outcomes. ${ }^{38,39}$

Genomic biobanking, which focuses on the storage of genetic material, has gained a foothold in the research culture of first world countries, with biobanks established in the 1990s in the USA, Europe, Ireland, Estonia, and the UK. ${ }^{40,41}$ While this is a relatively novel concept in Upper Middle Income countries (UMICs), it is well developed, with China first developing a biobank in 2006 and the China National Genebank in Shenzhen, established in 2011, being one of the largest in the world. ${ }^{42,43}$ Most Lower Middle Income countries (LMICs) surveyed, however, have suboptimal infrastructure and regulatory frameworks related to biobanking. ${ }^{43}$

Biodiversity is one of Africa's richest assets. It is therefore unsurprising that African genetic material is coveted by scientists internationally. While genetic and other biological material from communities are partially protected by Material Transfer Agreements, biopiracy (the act of directly or indirectly taking undue advantage of research participants and communities in global health research) has a long and contentious history in Africa. This has occurred as recently as the West African Ebola outbreak from 2014 to 2016 when thousands of biological specimens left the continent without consent. ${ }^{14,44,45}$ While multinationals and foreign academics and institutions profit from Africa's biodiversity, there is minimal benefit sharing with local communities and minimal acknowledgment of local researchers and academics. ${ }^{14,24,46}$

The H3Africa project (Human Health and Heredity, Africa), funded jointly by the U.S. National Institutes of Health (NIH) and the Wellcome Trust, has begun the 
process of biobank development in Africa. While the aim is to enhance capacity development by encouraging African scientists to establish biorepositories in African countries, including South Africa, data and samples still leave the continent to collaborators abroad with broad consent and with minimal, if any, benefit sharing with local communities. $^{24,47-49}$ Broad consent is controversial because participants consent to current and unknown future use of their samples, usually with approval of a Research Ethics Committee. However, this unknown future use may occur in a foreign country outside Africa. Some argue that unknown future use cannot be informed. While H3Africa regards capacity development as benefit sharing, local communities do not stand to benefit from the donation of their samples in more explicit ways. ${ }^{48,50}$ The use of broad consent has introduced a new discourse in health research, with commensurate ethical concerns, especially where broad consent is required to facilitate the continuing exodus of samples and data. ${ }^{14,24,48,49}$

While the large sample size and resultant predictive value of genomic analysis herald major advances in the assessment of community wide health, it also presents unique ethical challenges, in that the sampling may not be directly beneficial to the individual, and it may lead to the stigmatization of recognizable communities due to their association with major disease, such as with Tay Sachs and Ashkenazi Jews or Sickle Cell Anemia and Black African populations. ${ }^{45,51,52}$ The ethical concerns around genomic biobanking are exacerbated in the absence of authentic CE strategies.

\section{The State of CE, a Problem of Tokenism}

While CE is an overwhelmingly positive component of research and is closely intertwined with a robust consent process, it is often seen in isolation and can be considered mere ritual, rubberstamping, window-dressing, or tokenism, riddled with power and technical imbalances, placation, dishonesty, poor representation, and a lack of legitimate control over the research processes by the community. ${ }^{15,16,44}$ Arnstein, in 1969, articulated the various levels of citizen participation, in the context of city planning, conceptualized as rungs on a ladder, ranging from nonparticipation (manipulation, therapy, and informing), degrees of tokenism (informing, consultation, and placation), to degrees of citizen power (partnership, delegated power, and citizen control). ${ }^{53}$

Tokenism is widely evident in CE in research settings where asymmetrical power relationships exist between researchers and participants and where perfunctory or symbolic efforts are made to tick boxes without authentic engagement and benefit sharing. Late involvement with communities, superficial engagement, or engagement as an afterthought is regarded as tokenistic CE. ${ }^{54}$ In South Africa, the most common type of $\mathrm{CE}$ involves the formation of Community Advisory Boards (CABs). While necessary as a component of $\mathrm{CE}, \mathrm{CABs}$ are by no means sufficient. $\mathrm{CE}$ in general and $\mathrm{CABs}$, more specifically, are often criticized as being ancillary to research. ${ }^{55}$

Similarly, resources allocated to the development and management of $\mathrm{CE}$ and $\mathrm{CABs}$ tend to be limited and are often the first to be cut from study budgets when research priorities are considered. In keeping with resource limitations, it is important to be explicit about what remuneration, if any, can be expected by members of the CABs. It is standard practice in South Africa that $\mathrm{CAB}$ members are reimbursed for attendance at meetings. This has the potential to attract unemployed community members who perceive participation as a source of income. Occasionally, the lines between $\mathrm{CE}$ and reimbursement may become blurred. This was our experience during the production of our biobanking video, when $\mathrm{CAB}$ members participating in the video demanded payment as professional actors. ${ }^{56}$

Reddy et al. identified four themes as important in the establishment of CABs, including a definition of purpose, membership and representation, power and authority, sources of support, and independence. ${ }^{57}$ There are three recommended methods by which CABs may be constituted. There is purposeful selection, where the members are chosen from groups interested in the project. A more democratic process entails election of members, while a mixed model combines aspects of purposeful and democratic processes. ${ }^{58}$ While CABs are constituted to represent the values and concerns of their communities, appointment of individual members may be based on accessibility and convenience rather than communal nomination. This may result in a lack of representation of those with time, resource, and transport limitations, including the truly destitute and poor, while the lack of veto power over research suggestions and approaches may lead to them serving primarily as sounding boards.

Notwithstanding the limitations of CABs, they have been shown to serve as intermediaries between the research team and potential or actual research participants. They often educate community members clarifying understanding to prevent therapeutic misconception. $9,13,59,60$

Despite the importance of $\mathrm{CE}$ in general and in genomic biobanking in particular, there is a dearth of research on this topic in resource-poor settings. ${ }^{32,61,62}$ Neither is there a suitable CE model. Consequently, we set out to develop a model based on our own research experiences in CE over the past 15 years, a literature review and empirical research in this field in a South African setting over the past 4 years. This article describes the culmination of our project on the ethical, legal, and social issues related to genomic biobanking.

\section{Development of the TRUCE Model}

The Centre for Medical Ethics and Law (CMEL), at the Faculty of Medicine and Health Sciences, Stellenbosch University, South Africa, was awarded an NIH research grant to explore the ethical, legal, and social issues involved in Genomic Biobanking and CE in 2015. This project was conducted in conjunction with a biobank established at the neighboring academic Tygerberg Hospital. After Research Ethics Committee (REC) approval for the project was granted, the CE process began with broad SE. Early conversations began with members of the Biobanking $\mathrm{CAB}$ and entrenched biobank staff, researchers, clinicians, REC members and biobank donors, research participants, and patients. The TRUCE model was developed based on insights we gathered, while implementing a number of empirical studies and $\mathrm{CE}$ activities. See Table 1 for highlights of these activities.

The TRUCE model, described below, is proposed as a model strategy for effective $\mathrm{CE}$ across all stages of a research project. While the contexts and limitations of each study will differ, following this model is likely to guide CE that is measurable, reliable, and relevant. Each step of the model is framed in the context of community consultation, which is a hallmark of Ubuntu. The TRUCE model consists of eight steps (Fig. 1): 
Table 1. Highlights of Empirical Research and Community Engagement Activities ThAT INFORMED THE TRUCE MODEL

$\mathrm{CAB}$ consultation

Educational video created

Script developed with input from all stakeholders

Pamphlets created

Content presented in lay language

Engagement with Department of Education and facilitation of workshops

Co-creation of new genetics curriculum

Activity book developed

Continued dissemination

Empirical research
The Biobanking $\mathrm{CAB}$ and staff were consulted to elicit their views on $\mathrm{CE}$. A significant finding in this empirical work was the emergence of different definitions of community. This has informed the development of the TRUCE model and is detailed in step 1 of the model ${ }^{14,24}$

An educational video was developed in conjunction with $\mathrm{CAB}$ members as well as other research stakeholders-nurses, scientists, and clinicians working within the biobank. After a series of meetings and discussions, a script was developed with input from all stakeholders. A Whatsapp group was created specifically for communication with community members who contributed to the script. Participants in the video comprised CAB members, biobank staff, and medical students. Professional actors were deliberately not used to ensure a more realistic portrayal of the biobank and its processes. This video has been widely distributed and is available on YouTube. ${ }^{80}$

Multiple pamphlets discussing biobanking, genetics research, and genomics research were created by members of the $\mathrm{CAB}$, community workers, scientists, and communication experts, in conjunction with the research team. Early engagement in the development of these tools was appreciated by community members. This is elaborated on in step 3 of the TRUCE Model. The text was written in lay language and at this stage, it was clear that there were no words for genes in local languages. For English-speaking participants, the understanding of genes was poor even in young participants who had received a high school education. There was a perception among participants that although biology was taught well at their schools, genetics teaching was suboptimal. This prompted an engagement with senior educationalists in the South African Department of Education, who in turn facilitated a workshop with 100 high school biology teachers in the Western Cape. During this workshop, comprising both interactive lectures and informal discussion, important insights were shared in an attempt to cocreate a new genetics curriculum for high school learners. The co-production of knowledge is detailed in step 5 of the TRUCE Model. An activity book on genetics and genomics was developed with similar broad input and workshopped with high school Life Sciences learners. Feedback from this community of learners and teachers was used to improve and edit the books.

Both the pamphlets and the video continue to be disseminated broadly, to every reachable stakeholder. Return of results of the project is discussed further in step 8 of the TRUCE Model.

Empirical research was conducted, comprising interviews with experts, ${ }^{14,24}$ research participants, CABs, and other stakeholder groups $\left(^{36}\right.$ and unpublished data).

CAB, community advisory board; CE, community engagement; TRUCE, Tygerberg Research by Ubuntu for Community Engagement Model.

(1) Use social mapping to identify potential communities.

(2) Set the scope of CE and plan accordingly.

(3) Approach communities early.

(4) Co-create strategies for CE.

(5) Reinforce co-ownership of knowledge production.

(6) Recruit and engage communities at each stage of research.

(7) Evaluate, reflect, and adapt CE strategies.

(8) Discuss and disseminate results.

\section{Use social mapping to identify potential communities}

The concept of CE needs to begin with an assessment and appreciation of what a "community" is. MacQueen discusses the five defining characteristics of a community as follows: locus, sharing, joint action, social ties, and diversity. ${ }^{63}$ It is important to recognize that there are communities of interest and communities of identity, and that each person may be involved in a multitude of communities based on their age, gender interests, or circumstances. ${ }^{64}$ This is analogous to the difference in motivation and expectation of patient groups in comparison with public groups, with the former advocating out of personal experience and for quality of medical services, with the latter advocating out of public good and for equity of medical services. ${ }^{5,65} \mathrm{CE}$ needs to involve a multitude of relevant forms of engagement, to ensure that this heterogeneity of community is accounted for.

There are multiple potential communities of interest that are specific to age, race, gender, religion, or other demographic qualifiers. Therefore, they should be approached in a multifactorial way, identifying whether you are dealing with a geographic or demographic community as opposed to a community of purpose. ${ }^{63,66,67}$ The latter often includes patients and potential participants. These communities may develop and evolve over time, and this should be accounted for. In an empirical research study of our Ethical, Legal and Social Issues of Genomic Biobanking project, participants defined their community as one due to geographical commons $^{47}$ (unpublished data). People in the community were "those living within close geographical proximity." Some had the perception that their community stretched further to 


\section{$\mathrm{T} \quad \mathrm{R}$}

TYGERBERG RESEARCH BY UBUNTU FOR

COMMUNITY ENGAGEMENT MODEL
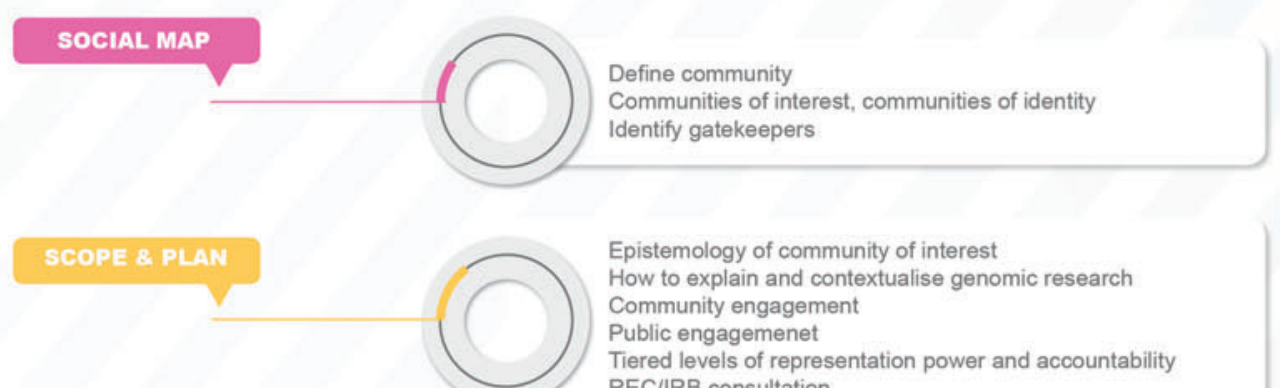

Epistemology of community of interest

How to explain and contextualise genomic research

Community engagement

Public engagemenet

Tiered levels of representation power and accountability REC/IRB consultation

$\begin{aligned} & \text { APPROACH } \\ & \end{aligned} \mid \begin{aligned} & \text { Engagement should begin early } \\ & \text { Before research question formulation } \\ & \text { Pre-existing beliefs } \\ & \text { Negotiate benefit sharing } \\ & \text { Gauge expectations } \\ & \text { Show respect }\end{aligned}$
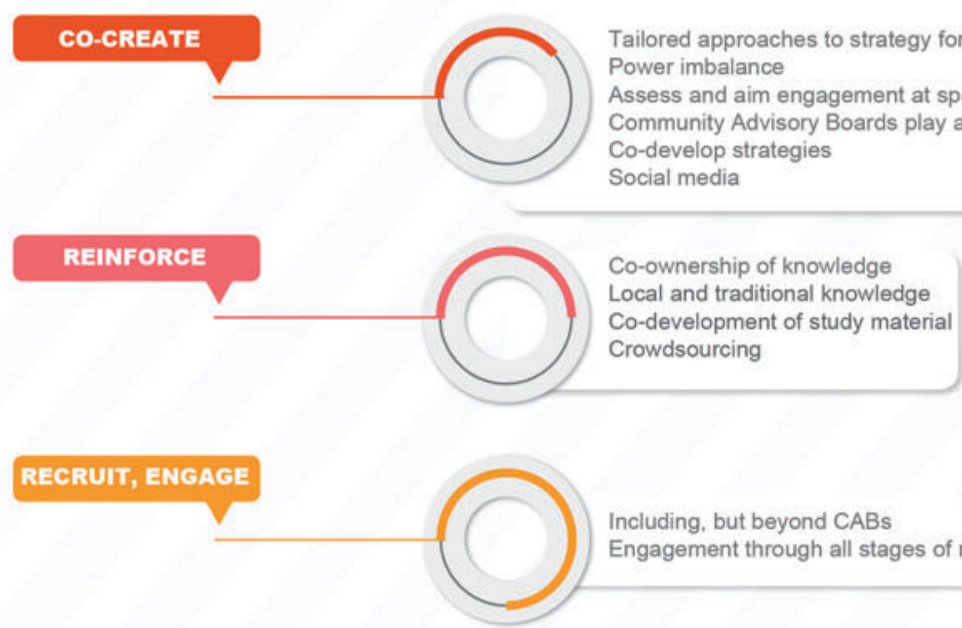

Including, but beyond CABs

Engagement through all stages of research

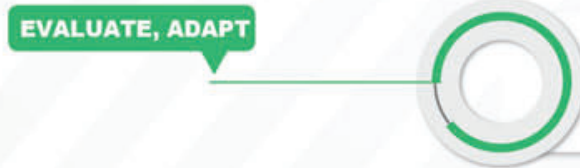

mpact and effectiveness of CE engagement Mixed methods - key performance indicators

Cross-triangulation of data

Constant dynamic ada

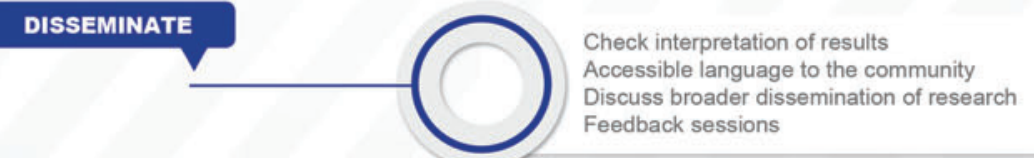

FIG. 1. The TRUCE Model. TRUCE, Tygerberg Research by Ubuntu for Community Engagement Model. Color images are available online. 
those in the same church, and to their family and circle of friends, "regardless of location."

This is in keeping with the concept of Ubuntu, where neighbors are viewed as members of the extended family with interdependent co-existence. ${ }^{68}$ According to Bongani Finca, "we don't live in isolation, we live in a community". ${ }^{29}$ At the Tygerberg Biobank, the community of potential donors are the patients who live closest to the hospital and who fall into the catchment area of the health service.

Engaging a variety of stakeholders, including at the community level, helps to articulate a shared theory of change and drawing of social and network maps. Social mapping can provide valuable guidance for researchers to identify what strategies would work best in a given research context. Social mapping is a visual method of illustrating the relative location of households and the distribution of different types of people by specifiers such as gender; age; ownership of land; and literacy level, together with the social structure and institutions of an area. An analysis of these data will help define communities of relevance to the proposed research. In our empirical work, respondents described three different layers of SE: community level, peer level, and high level. Community-level engagement included potential participants, CABs, and field workers, and peer-level engagement included researchers, biobankers, and scientists, while high-level engagement included government officials, funders, and policy makers. ${ }^{24}$ Comments elicited from a peer-level stakeholder group was as follows: "When we export material from SA or from underdeveloped countries to the developed countries, we have no control over its eventual fate. Are we being used as experimental collection depots for the third world?",14 Although education of each stakeholder layer is important, education of the community layer is most challenging, due to the complexity of the research and educational levels of stakeholders in this layer. ${ }^{24}$ Consequently, the TRUCE Model focuses on $\mathrm{CE}$ at this level.

Often, senior members of the communities, such as chiefs, serve as gatekeepers, while others regarded as community leaders include religious leaders, teachers, influential people, celebrities, politicians, or council members such as the San Council in southern Africa. ${ }^{1,20,69,70}$ Approaching gatekeepers to communities must only be seen as an early step in the CE process. Individual members of the community retain the right to participate in the engagement process as they choose. With the San community in South Africa, where genetic and genomics research occur frequently, researchers may only approach the community after the San Council has agreed. Subsequently, the community discusses the research plan and votes about whether they should participate in the study or not. Each member of the community then provides consent individually. Research can then proceed in keeping with the San code of ethics. ${ }^{62}$ A judicious choice of methods for bringing people together, and supporting their debate and decisions, depends upon the drives and characteristics of those involved, particularly their degree of enthusiasm, experience, and motivation. ${ }^{71}$

\section{Scope of CE and planning}

The scope of CE needs to be determined at the onset. This will depend on the type of research being conducted as well as demographics of the relevant communities. ${ }^{72}$
Once communities are defined, establishing the scope of CE will determine whether specific communities will be approached or whether a wider type of public engagement needs to occur. It is important to keep the scope of purpose and objectives in line with relevant legislation, regulations, and overarching aims of the research institution. ${ }^{72}$ Communication with RECs is critical at this stage. It may be necessary to seek approval from the REC to conduct formative research in the community ahead of the actual research project. In large projects, approval of the main research study may take several months. In this time, CE can start at a generic level, testing perceptions around important concepts planned for the larger study. In the case of genomic biobanking, testing the acceptability of broad consent may be relevant using hypothetical scenarios. Empirical research conducted with 200 participants at six clinical trial sites in South Africa indicated that almost half the sample did not support broad consent. ${ }^{50}$ Likewise, assessing understanding of DNA and genes may be relevant (Asian Bioethics Review + unpublished data). ${ }^{47}$ In other projects, like emergency trauma research, it may be necessary to assess perceptions on delayed consent before conducting the actual study.

How do we assess the degree of understanding or approach to systems of knowledge prevalent in the relevant community? Genomic biobanking involves particularly complex scientific concepts that many communities, even in developed world contexts, do not fully understand. Our empirical work with patients revealed that the concept of genetic inheritance is often best approached by creating a family tree and noting degrees of similarity between familial individuals as well as diseases prevalent in more than one member (unpublished data). It is also important to establish how each community approaches knowledge. While some communities are more influenced by the scientific method, others place more value in the opinions of elders and established cultural traditions. ${ }^{35,59,73,74}$

Implementation research considers a range of conceptual, ethical, and practical issues relevant to CE, while determining how the study will ensure adequate and equitable representation from underserved communities. ${ }^{21}$ Applying systems thinking methods and tools assists in understanding and analyzing complex system dynamics and relationships associated with specific interventions. Understanding the communities' concerns, cultural sensitivities, religious beliefs, barriers to participation, and potential misperceptions, as well as assessing the variations in resources, education levels, and purpose are relevant to the prospective scope of the CE process (unpublished data). ${ }^{58}$

In planning how to encourage research participation, purely altruistic appeals can be considered manipulative and can undermine the respect required in research endeavors. Solidarity and reciprocity are alternative prosocial appeals that have been shown to have more relevance and efficacy in encouraging participation in genomic research. ${ }^{75}$

\section{Approach communities early}

Approaching community stakeholders must be undertaken in a way that is appreciative of their expertise as well as their availability. ${ }^{2}$ Ideally, engagement should start before formulation of the research question so that health needs and health priorities of the community can be 
established. Furthermore, early engagement allows researchers to assess community assets in terms of knowledge, skills, and resources. This baseline assessment allows the team to augment existing resources. Participants in one of our studies described genes as 'something in the blood' associated with inheritance, but also indicated that they "don't know where they are." 50 This guided the development of educational pamphlets, the educational video, and future interviews. Early engagement also informs the research team of pre-existing cultural beliefs. A CAB member indicated, "Some people would perhaps say 'No I can't, what are my ancestors going to say' so they have to go and ask the elders first if they may do it." Another CAB member indicated that, "You find native people like me, isiXhosa people, who find blood to be very sacred. If you have something of your own being in the possession of someone else, there's a danger involved, you see.' Unmasking these cultural beliefs occurred in our early conversations with potential participants.

Respect is an important principle in CE and early CE is a sign of such respect. The San community of South Africa has a specific code of ethics for research, which needs to be respected by all potential researchers. ${ }^{70}$ This level of respect should be extended to all members of relevant communities. Building a relationship with a community must be a primary aim in and of itself, with each study building on the work done by previous studies. The researcher, as a stranger to the community, needs to allow and facilitate the community getting to know them and becoming comfortable with their presence. This can be aided by advocating for the confirmation of the researcher's integrity as well as the integrity of their research institutions before eliciting participation from community members. It is important to be explicit about the intended and actual use of data, as communities in resource-restricted contexts are often wary of commercialization and exploitation of their data. If there is any prospect of commercialization, this must be made explicit. ${ }^{62,76}$ Communities must be empowered to negotiate benefit sharing early in the process of engagement. This is a requirement in the San Code of Ethics and applies to research on the San people in Southern Africa, but is also a general research ethics obligation based on the principle of justice. Those who bear the burdens of research should also benefit. Apart from providing assurance around privacy and the limits of confidentiality in genomics research, participants must be made aware of their right to withdraw consent for storage and use of their data and specimens at any time. Researchers must be trained specifically in communicating respectfully, resolving conflict with maturity, and responding to concerns relating to local contexts, and embedded social relationships. Ubuntu supports an approach toward a community of reciprocal relationships, considered keystone to socially accountable medical practice and has been described by participants as the foundational concept supporting a positive interaction between the reciprocal relationships and the social determinants of a community's health. $^{27}$ Transparency is an important concept in early engagement. It is important to initiate an open and transparent discussion of what products and services will be made available to study participants to ameliorate the potential for participants to decide to participate in research based on inaccurate expectations of ancillary care, postresearch care, and therapeutic misconception. ${ }^{2}$ Since mon- etary incentives have not been shown to increase recruitment, this needs to be discussed in the context of existing guidelines. ${ }^{61}$ Early engagement is a sign of respect as the community is then aware that they are not being involved as an afterthought. A culture and relationship of trust can be built and maintained by demonstrating the value the research team sees in members of the community, beyond their involvement in any specific study. The consent document itself needs to be created with the direct, and complete involvement of the community, often by way of their representatives, both on and off relevant CABs. The value in this approach is that the concepts, expectations, approaches, interventions, and prospective value or risks of the research can be fully understood by potential participants. This process goes beyond simply translating the language, as certain concepts in genomics and biobanking cannot be directly translated to African languages. These terms may be contextually explained allegorically to optimize understanding. ${ }^{35,69}$ Discussions around return of results is part of benefit-sharing. Where genetic and genomic testing are part of a project, return of results or lack thereof is critical. Prior studies have shown that individuals are more likely to enroll in large genomic studies and biobanks when feedback on Individual Research Results (IRRs) was offered, although not everyone is comfortable in receiving every type of result. ${ }^{77}$ Many participants appreciate the opportunity to set preferences, or filters, with regard to the types of IRRs. The potential benefits may include screening for disease, as well as forming reproductive decisions. Potential negatives include anxiety, confidentiality being problematic, career or insurance repercussions, and the risks regarding the penetrance of certain disease, not being well understood. When data or samples are stored in an anonymous format, the challenges with return of results must be explained. Communities must not be made to feel coerced into involvement, and it must be made very clear that their choice to participate, or, perhaps more importantly, not to participate, will in no way influence any aspect of their ordinary medical care. ${ }^{14,24,47}$ Problems may arise in terms of how consensus can be reached in a community with various cultural and hierarchical views. This process requires mutual understanding of the foundational aspects of research designs, taking into account the vastly differing epistemological bases of the community and research team. ${ }^{76}$ Researchers need to use various methods in advancing available evidence to achieve this mutual understanding. These methods may include workshops, poster campaigns, and/or social media, emphasizing the provision of regular updates to the community. This consensus is particularly important when creating consent documents. It is clear that early engagement enhances the consent process with mutual benefit to researchers and participants.

\section{Co-create strategies for CE}

The strategy used to approach a specific group or community must be contextualized. In traditional African communities, approaching the chief and getting his consent, approval, and aid are beneficial. Engagement strategies must be tailored to the educational level of different communities. When we discovered that basic knowledge around genes and genetics was suboptimal, we started to engage with the Department of Education and biology teachers in the 
Western Cape. High school students can be approached through teachers, the School Governing board, and parents or caregivers. The youth can be best approached through social media, given their ubiquitous involvement in this digital space. ${ }^{62,78-80}$

Although CABs may play a role in the recruitment, enrolment, and follow-up participants, communities must be engaged in a manner that respects how they wish to be approached.

Where required, training and technical support for community members will be necessary to avoid a power imbalance that may render the $\mathrm{CE}$ process merely consultative, which, if not accounted for, may engender discontent and distrust in the community. Likewise, training of research staff to develop the necessary skills to engage effectively with communities is a critical pre-requisite in CE. Communication as well as negotiation skills and conflict resolution are central to building social capital.

\section{Reinforce the co-ownership of knowledge production}

Knowledge co-production and ownership can be understood as an interactive and dynamic endeavor of multiple actors where conventional epistemological realms and roles of different actors are blurred. ${ }^{80}$ This draws attention to the dynamic changes that occur in understandings, norms, beliefs, and practices, where knowledge co-production serves as a source of learning. ${ }^{81}$ The co-ownership of knowledge that flows from the research should be emphasized as scientists and academics often take a disproportionate share of the credit for research outcomes. Co-ownership brings together local and traditional knowledge with scientific knowledge and authentically acknowledges the role played by communities in knowledge production. This is important to flatten hierarchies and reduce imbalances in power and privilege that are a hallmark of researcher-participant relationships. In our Genomic Biobanking project, various stakeholders, including CAB members, provided input into the development of educational pamphlets and the video script from the start. Some members of stakeholder groups participated in the videos as "actors." 82 The actual engagement with the community, relevant metrics for success, opportunities for engagement, and adjustment of aspects of the study must be mutually decided upon through rigorous debate and involvement with relevant $\mathrm{CE}$ representatives. The co-creation and democratization of knowledge involve incorporating all relevant stakeholders, including patients, doctors, researchers, and policy makers, in all phases of a research project from research design, and proposal appraisal, to patient recruitment, through to the interpretation of results and subsequent implementation of research outcomes. ${ }^{81}$ This process, aided by relevant crowd sourcing, allows for knowledge and services to be better adjusted to the needs and preferences of involved communities. Crowdsourcing involving outsourcing problems and tasks to a crowd, often through the internet and relevant technology, is particularly useful, cost effective, and rapid in health research. ${ }^{83}$ This leverages the collective intellect of online communities for specific goals and has been shown to improve intervention development and evaluation, as well as enhancing the facilitation of communication. ${ }^{84}$ The empowerment of communities in research should be a primary aim, with each subsequent project building upon this. A focus on the creation of varied, but equal roles in groups and subgroups, which build upon the skills of the individual members of the community, is vital. These groups, once sufficiently knowledgeable over the research process, may in turn be empowered as research oversight committees, working alongside Institutional Health Research Ethics Committees (HRECs) to govern standards of research conducted in their communities. ${ }^{44}$

\section{Recruit and engage communities at each stage of research}

While CABs can be constituted in research studies to assist in managing the expectations of participants, this should not be the only form of CE. CABs have many roles and clear messaging regarding the research processes; benefits to potential participants and the community, with an explanation and discussion of the commensurate risks, are important. ${ }^{13,47,57}$ The roles of a CAB include, inter alia, the protection of community interests, while advancing research goals. In protecting community interests, they may focus on stopping exploitation and ensuring community benefit, while providing substantive input (from inception to conclusion) and building capacity by way of a culture of human rights and empowerment of communities. Research goals can be advanced by providing bilateral information sharing, acting as a public relations buffer, and assisting with recruitment. There is a feeling that CABs should educate the community on the study as well as serve as gatekeepers to the community, assisting researchers in gaining access into the community, but not engaging in active recruitment to avoid creating a sense of conflict. While CABs should not be directly involved in recruitment, they play an invaluable role in facilitating the informed consent process, helping in the development of materials that explain the study to participants, and representing the participants' concerns to the researchers. ${ }^{85} \mathrm{~A}$ researcher indicated in one of our studies that "Consent forms should evolve in consultation with $\mathrm{CABs}$ so that the consent form can actually contain the information that would be really important from the patient and community perspective." He went on to say that, "At the moment the consent form is the product of the researcher/research team and biobank perspectives...it contains literature from an ethics and legal perspective...it may not be important from the patient's perspective." 14

$\mathrm{CABs}$ ought to advocate for full disclosure of the risks and benefits of the study to their constituent communities. It is important that $\mathrm{CABs}$ maintain their independent advisory voice, ensuring that their input into the research process is fair and constructive, respects the scientific process, and is in the best self-identified interests of community members; in this way, it is worthwhile for the CABs to account not just to the research team, but to the community as well. ${ }^{58} \mathrm{CE}$ is fundamentally about building relationships, and how this relationship is built will determine the successful implementation and sustainability of research projects, including genomic biobanking. ${ }^{86}$ Potential participants should be invited to voluntary information sessions on the concept of genomic biobanking in general, and the study, specifically as part of the screening process. In our empirical research, discussions regarding the family tree were useful to conceptualize genetics and inheritance, and information was 
supplemented with pamphlets and educational videos. It is worthwhile to use several tools and media platforms to engage the community on a continuous basis and in an interactive manner through community newspapers, posters, radio, and social media throughout the life cycle of the research project. Historically, the focus of engagement has been in the earlier stages of clinical trials, such as protocol explanation and recruitment, with significantly less engagement in the later stages involving the results and dissemination thereof. Future CE must be explicit in not perpetuating this mistake. $^{87}$

\section{Evaluate, reflect, and adapt CE strategies}

The impact and effectiveness of CE need to be assessed to establish legitimacy within the broader scientific community. ${ }^{47}$ Mixed methods analyses can be useful in evaluating the effectiveness of the CE approach, while the crosstriangulation of data may provide deeper information around the nature of relevant research relationships. ${ }^{3,36}$

Key performance indicators must be established at the outset, from thematic analysis, including systematic and specific indicators to be evaluated by way of continuous, dynamic, mixed methods. Key issues identified include a limited conceptualization of $\mathrm{CE}$, poor quality of methods reporting, unclear context validity of studies, poor reporting of context and process, enormous variability in the way impact is reported, little formal evaluation of the quality of involvement, limited focus on negative impacts, and little robust measurement of impact. ${ }^{88}$ The GRIPP- 2 checklist is an example of a way of addressing these. ${ }^{10,89}$ The GRIPP2Long Form is suitable for studies where patient and public involvement (PPI) is the primary aim, while the GRIPP2Short Form is suitable for studies where PPI is a secondary focus. ${ }^{10}$ Another example is Khodyakov's CE in Research Index, which covers each aspect of the research process, and whether the community was actively engaged, consulted, or did not participate. ${ }^{90}$ The CE strategies employed should be adapted dynamically within a study, as well as for each subsequent study. Realistic evaluation can assist in this adaptation of strategies. This form of evaluation aims to find out the contextual factors that make interventions effective, to understand why interventions work in some conditions, but not others. ${ }^{4}$ These evaluations are unique in their approach as they begin with a theory about how actions and mechanisms work in each particular context to create a desired outcome. This process is repeated in an iterative manner that aids in generating a hypothesis about what specific aspects of an intervention affect change, and specifically which groups may benefit. These results are fed back into the process to create a series of studies that each advance the next. $4,78,91$

\section{Discuss and disseminate results}

Following review of the research by the research team, the analysis and dissemination of the results to the community and specific stakeholders is an important final step. This should follow relevant communication channels developed during the $\mathrm{CE}$ process using easily understandable language. ${ }^{92}$ Discussing the research results is important to check if the interpretation of the research team was correct. Involving the $\mathrm{CAB}$ or other community members who participated in the research process, in screening lay publications and in advising the research team, is important. ${ }^{93}$ These stakeholders could also help to facilitate understanding in the community by contributing to the feedback sessions. The research team should also, at this point, discuss the plans for broader dissemination of the research with the community, paying particular attention to accrediting the community for the role they have played in the research, and the way in which they would like to be acknowledged in subsequent publications. In acknowledging communities in research publications, it is important not to inadvertently offend or stigmatize them. For example, in research on the San community, the San Council has articulated a preference for use of San and Khoi separately, but not Khoisan (personal communication). Stigmatization of communities with HIV or TB can also occur, especially through publication in the media where specific geographical areas become associated with specific diseases.

\section{Limitations of Our Model}

Our model is designed for a developing world, urban setting, and therefore the approaches may not be broadly generalizable to the developed world, or to rural settings. While we made a concerted effort to appraise all available literature on this topic from medical, allied health sciences, and social sciences, we did not conduct a systematic review or meta-analysis of data available as both had been done fairly recently. We did, however, consult with prominent researchers on this topic, and do not believe that the lack of a systematic review made a material difference on our approach. There still exists a dearth of literature examining biobanks in Africa specifically, and developing countries in general, which makes creating a model especially difficult. While the empirical work conducted for the ELSI Genomic Biobanking project contributed to the development of the TRUCE model, the broad principles implicit in the model can be extrapolated to other types of research. The model proposed in this study remains to be evaluated in a future project.

\section{Recommendations}

Implementing the TRUCE CE Model is resource dependent. Consequently, the following recommendations are proposed. Making CE a funding requirement by including it in funding proposals and including it as a budget line item in grants is necessary where research is conducted among communities. Specific training in CE for research teams is essential. Furthermore, RECs/IRBs have a role to play in requiring a $\mathrm{CE}$ plan for every study that is community based, and scientific journals ought to require a paragraph on $\mathrm{CE}$ in publication of relevant research projects. Much of this can be ensured by moving $\mathrm{CE}$ from a guidance requirement to a regulatory requirement, emphasizing that it is a critical component of a robust consent process in research and that it ought to be embedded within research projects, where applicable. Communities in LMICs must not be left behind in the Fourth Industrial Revolution. Instead, we need to explore ways of harnessing digital technology to augment CE through wearables, mobile technology, access to data, and bandwidth. Such technology will make dynamic consent and durable engagement with tissue trusts and biobanks possible in low resource contexts. 


\section{Conclusion}

$\mathrm{CE}$ is critical in genomic biobanking in a resourceconstrained setting where there is suboptimal understanding of genetics, genomics, and biobanking, yet it is an often neglected component of research. There is a significant gap between the theory of $\mathrm{CE}$ and application to conduct of research. There are also significant disparities between how $\mathrm{CE}$ is approached in resource-rich and resource-poor countries. The digital divide is expanding and technology must be harnessed to improve CE. It is hoped that the TRUCE model will facilitate the bridging of that gap by integrating the Ubuntu-derived concept of humanism and respect for dignity of research participants globally.

\section{Acknowledgments}

The authors would like to thank and acknowledge the staff of the CMEL, as well as current and previous associates who contributed to the empirical research upon which this model is developed. These include Ciara Staunton, Paulina Tindana, Eric Juengst, Melany L. Hendricks, Claire E de Koker, Lorrein S Muhwava, and Fatima Bassa. We would also like to thank the National Institutes of Health in connection with a research grant awarded to explore the ethical, legal, and social issues involved in Genomic Biobanking and Community Engagement.

\section{Author Disclosure Statement}

The authors have no relevant financial, personal, political, or academic conflicts of interest to declare.

\section{Funding Information}

National Human Genome Research Institute of National Institutes of Health. Award number UO1HG008222.

\section{References}

1. George AS, Mehra V, Scott K, Sriram V. Community Participation in Health Systems Research: A Systematic Review Assessing the State of Research, the Nature of Interventions Involved and the Features of Engagement with Communities. PLoS One 2015;10:e0141091.

2. Newman PA, Rubincam C. Advancing community stakeholder engagement in biomedical HIV prevention trials: Principles, practices and evidence. Expert Rev Vaccines 2014;13:1553-1562.

3. Snape D, Kirkham J, Preston J, et al. Exploring areas of consensus and conflict around values underpinning public involvement in health and social care research: A modified Delphi study. BMJ Open 2014;4:e004217.

4. Staley K, Buckland SA, Hayes H, Tarpey M. 'The missing links': Understanding how context and mechanism influence the impact of public involvement in research. Health Expect 2014;17:755-764.

5. McCoy MS, Jongsma KR, Friesen P. National standards for public involvement in research: Missing the forest for the trees. JME 2018;44:801-804.

6. NHS. INVOLVE. Available from: www.invo.org.uk.

7. Institute P-COR. PCORI Washington, DC. Available from: www.pcori.org.

8. Forsythe LP, Ellis LE, Edmundson L, et al. Patient and stakeholder engagement in the PCORI pilot projects: De- scription and lessons learned. J Gener Intern Med 2016;31: 13-21.

9. Harris J, Croot L, Thompson J, Springett J. How stakeholder participation can contribute to systematic reviews of complex interventions. J Epidemiol Commun Health 2016; 70:207-214.

10. Staniszewska S, Brett J, Simera I, et al. GRIPP2 reporting checklists: Tools to improve reporting of patient and public involvement in research. BMJ 2017;358:j3453.

11. Staunton C, Moodley K. Challenges in biobank governance in Sub-Saharan Africa. BMC Med Ethics 2013;14:35.

12. George AS, Scott K, Mehra V, Sriram V. Synergies, strengths and challenges: Findings on community capability from a systematic health systems research literature review. BMC Health Services Res 2016;16(Suppl 7):47-59.

13. Manafo E, Petermann L, Vandall-Walker V, Mason-Lai P. Patient and public engagement in priority setting: A systematic rapid review of the literature. PLoS One 2018;13:e0193579.

14. Moodley K, Singh S. "It's all about trust": Reflections of researchers on the complexity and controversy surrounding biobanking in South Africa. BMC Med Ethics 2016; 17:57.

15. Domecq JP, Prutsky G, Elraiyah T, et al. Patient engagement in research: A systematic review. BMC Health Serv Res 2014;14:89.

16. Price A, Albarqouni L. Patient and public involvement in the design of clinical trials: An overview of systematic reviews. J Eval Clin Pract 2018;24:240-253.

17. Madden M, Speed E. Beware Zombies and Unicorns: Toward Critical Patient and Public Involvement in Health Research in a Neoliberal Context. Front Sociol 2017;2:7. DOI: $10.3389 /$ fsoc.2017.00007.

18. Ives J, Damery S, Redwod S. PPI, paradoxes and Plato: Who's sailing the ship? J Med Ethics 2013;39:181-185.

19. Tapp H, White L, Steuerwald M, Dulin M. Use of community-based participatory research in primary care to improve healthcare outcomes and disparities in care. J Comp Eff Res 2013;2:405-419.

20. Unertl KM, Schaefbauer CL, Campbell TR, et al. Integrating community-based participatory research and informatics approaches to improve the engagement and health of underserved populations. J Am Med Inform Assoc 2016;23:60-73.

21. Glandon D, Paina L, Alonge O, Peters DH, Bennett S. 10 Best resources for community engagement in implementation research. Health Policy Plan 2017;32:1457-1465.

22. Emerson CI, Singer PA, Upshur RE. Access and use of human tissues from the developing world: Ethical challenges and a way forward using a tissue trust. BMC Med Ethics 2011;12:2.

23. Lucassen A, Montgomery J, Parker M. Chapter 16. Ethics and the social contract for genomics in the NHS. South Africa: South African Department of Health; 2017.

24. Staunton C, Tindana P, Hendricks M, Moodley K. Rules of engagement: Perspectives on stakeholder engagement for genomic biobanking research in South Africa. BMC Med Ethics 2018;19:13.

25. Gradinger F, Britten N, Wyatt K, et al. Values associated with public involvement in health and social care research: A narrative review. Health Expect 2015;18:661-675.

26. Heaven A, Brown L, Foster M, Clegg A. Keeping it credible in cohort multiple Randomised Controlled Trials: The Community Ageing Research 75+ (CARE 75+) study model of patient and public involvement and engagement. Res Involvement Engagement 2016;2:30. 
27. Green-Thompson LP, McInerney P, Woollard B. The social accountability of doctors: A relationship based framework for understanding emergent community concepts of caring. BMC Health Serv Res 2017;17:269.

28. Smith MK. Bringing Back Ubuntu. Acad Psychiatry 2016; 40:721-722.

29. Gade C. What is Ubuntu? Different interpretations among South Africans of African Descent. S Afr J Philosophy 2012;31:484-503.

30. Etzioni A. Authoritarian versus responsive communitarian bioethics. J Med Ethics 2011;37:17-23.

31. Nalubega S, Evans C. Participant views and experiences of participating in HIV research in sub-Saharan Africa: A qualitative systematic review. JBI Database System Rev Implement Rep 2015;13:330-420.

32. Ogunrin O, Woolfall K, Gabbay M, Frith L. Relative solidarity: Conceptualising communal participation in genomic research among potential research participants in a developing Sub-Saharan African setting. PLoS One 2018;13:e0195171.

33. Eze MO. What is African Communitarianism? Against Consensus as a regulative ideal. S Afr J Philosophy 2008; 27:386-399.

34. Mbiti J. African Religions and Philosophy. Nairobi: East African Education Publishers, Ltd.; 1969: 108-109.

35. Beaton A, Hudson M, Milne M, et al. Engaging Maori in biobanking and genomic research: A model for biobanks to guide culturally informed governance, operational, and community engagement activities. Genet Med 2017;19: 345-351.

36. Farmer J, Carlisle K, Dickson-Swift V, et al. Applying social innovation theory to examine how community codesigned health services develop: Using a case study approach and mixed methods. BMC Health Services Res 2018;18:68.

37. Johnson VA, Powell-Young YM, Torres ER, Spruill IJ. A systematic review of strategies that increase the recruitment and retention of African American adults in genetic and genomic studies. ABNF J 2011;22:84-88.

38. Collins SR. Big Data in the UK Biobank: Opportunity and Challenges. Gresham College: Youtube; 2014.

39. Bycroft C, Freeman C, Petkova D, et al. The UK Biobank resource with deep phenotyping and genomic data. Nature 2018;562:203-209.

40. Eisenhauer ER, Tait AR, Rieh SY, Arslanian-Engoren CM. Participants' Understanding of Informed Consent for Biobanking: A Systematic Review. Clin Nurs Res 2017: 1054773817722690.

41. Miller KL, Alfaro-Almagro F, Bangerter NK, Thomas DL, Yacoub E, Xu J. Multimodal population brain imaging in the UK Biobank prospective epidemiological study. Nat Neurosci 2016;19:1523-1536.

42. Li H, Ni M, Wang P, Wang X. A survey of the current situation of clinical biobanks in China. Biopreserv Biobank 2017;15:248-252.

43. Mendy M, Caboux E, Sylla BS, Dillner J, Chinquee J, Wild C. Infrastructure and facilities for human biobanking in low- and middle-income countries: A situation analysis. Pathobiology 2014;81:252-260.

44. Slack C, Wilkinson A, Salzwedel J, Ndebele P. Strengthening stakeholder engagement through ethics review in biomedical HIV prevention trials: Opportunities and complexities. J Int AIDS Soc 2018;21 Suppl 7:e25172.

45. Weijer C. Protecting communities in research: Philosophical and pragmatic challenges. Camb Q Healthc Ethics 1999;8:501-513.
46. World Health Organization. Report on the 2nd WHO Consultation on Biobanking: Focus on West Africa. Freetown, Sierra Leone: WHO; 2015.

47. Moodley K. Legitimacy, Trust and Stakeholder Engagement: Biobanking in South Africa. Asian Bioethics Rev 2017;9:325-334.

48. Dauda B, Joffe S. The benefit sharing vision of H3Africa. Dev World Bioeth 2018;18:165-170.

49. Tindana P, Molyneux S, Bull S, Parker M. 'It is an entrustment': Broad consent for genomic research and biobanks in sub-Saharan Africa. Dev World Bioeth 2019;19: 9-17.

50. Moodley K, Sibanda N, February K, Rossouw T. "It's my blood": Ethical complexities in the use, storage and export of biological samples: Perspectives from South African research participants. BMC Med Ethics 2014;15:4.

51. Myerowitz R, Costigan FC. The major defect in Ashkenazi Jews with Tay-Sachs disease is an insertion in the gene for the alpha-chain of beta-hexosaminidase. J Biol Chem 1988; 263:18587-18589.

52. Lorey FW, Arnopp J, Cunningham GC. Distribution of hemoglobinopathy variants by ethnicity in a multiethnic state. Genet Epidemiol 1996;13:501-512.

53. Arnstein SR. A Ladder of Citizen participation. J Am Inst Plann 1969;35:216-224.

54. Hoffmann AE, Hahn DL, Felzien M, LeMaster JW, Xu J, Fagnan LJ. Tokenism in patient engagement. Fam Pract 2016;34:290-295.

55. MacQueen KM, Auerbach JD. It is not just about "the trial": The critical role of effective engagement and participatory practices for moving the HIV research field forward. J Int AIDS Soc 2018;21 Suppl 7:e25179.

56. Staunton C, Abayomi A, Bassa F, Moodley K. Negotiating requests for reimbursement for community engagement: Challenges in developing an educational video for genomic biobanking research in South Africa. J Empir Res Hum Res Ethics 2019 [Epub ahead of print]; DOI: 10.1177/ 1556264619856223.

57. Reddy P, Buchanan D, Sifunda S, James S, Naidoo N. The role of community advisory boards in health research: Divergent views in the South African experience. SAHARA J 2010;7:2-8.

58. Mwinga A, Moodley K. Engaging with Community Advisory Boards (CABs) in Lusaka Zambia: Perspectives from the research team and $\mathrm{CAB}$ members. BMC Med Ethics 2015;16:39.

59. Lo Y-R, Chu C, Ananworanich J, Excler J-L, Tucker JD. Stakeholder engagement in HIV cure research: Lessons learned from other HIV interventions and the way forward. AIDS Patient Care STDs 2015;29:389-399.

60. Campbell MM, Susser E, de Vries J, et al. Exploring researchers' experiences of working with a researcher-driven, population-specific community advisory board in a South African schizophrenia genomics study. BMC Med Ethics 2015;16:45.

61. Tindana P, Campbell M, Marshall P, et al. Developing the science and methods of community engagement for genomic research and biobanking in Africa. Global Health Epidemiol Genom 2017;2:e13.

62. Tindana P, de Vries J, Campbell M, et al. Community engagement strategies for genomic studies in Africa: A review of the literature. BMC Med Ethics 2015;16:24.

63. MacQueen KM, McLellan E, Metzger DS, et al. What is community? An evidence-based definition for participatory public health. Am J Public Health 2001;91:1929-1938. 
64. Hashagen S, Scottish Community Development C; Models of community engagement [Glasgow]: Scottish Community Development Centre; 2002 Available from: www .communityplanning.org.uk/documents/Modelsofcommunity engagement.pdf.

65. Fredriksson M, Tritter JQ. Disentangling patient and public involvement in healthcare decisions: Why the difference matters. Sociol Health Illn 2017;39:95-111.

66. MacQueen KM, Alleman P. International perspectives on the collection, storage, and testing of human biospecimens in HIV research. IRB 2008;30:9-14.

67. MacQueen KM, Bhan A, Frohlich J, Holzer J, Sugarman J. Evaluating community engagement in global health research: The need for metrics. BMC Med Ethics 2015;16:44.

68. Mnyaka M, Motlhabi M. The African Concept of Ubuntu/Botho and its Socio-Moral Significance. Black Theol 2005;3: 215-237.

69. Chilvers J. Reflexive engagement? actors, learning, and reflexivity in public dialogue on science and technology. Sci Commun 2012;35:283-310.

70. Callaway E. South Africa's San people issue ethics code to scientists. Nature 2017;543:475-476.

71. Oliver S, Liabo K, Stewart R, Rees R. Public involvement in research: Making sense of the diversity. J Health Services Res Policy 2015;20:45-51.

72. Health Consumers Queensland. Developing a Consumer and Community Engagement Strategy: a Toolkit for Hospital and Health Services. Brisbane: State of Queensland, Queensland Health; July 2012.

73. Celi LA, Ippolito A, Montgomery RA, Moses C, Stone DJ. Crowdsourcing knowledge discovery and innovations in medicine. J Med Internet Res 2014;16:e216.

74. Stadler J, Dugmore C, Venables E, MacPhail C, DelanyMoretlwe S. Cognitive mapping: Using local knowledge for planning health research. BMC Med Res Methodol 2013;13:96.

75. Guttman N, Siegal G, Appel N, Bar-On G. Should Altruism, Solidarity, or Reciprocity be Used as Prosocial Appeals? Contrasting conceptions of members of the general public and medical professionals regarding promoting organ donation. J Commun 2016;66:909-936.

76. Hall MA, King NMP, Perdue LH, et al. Biobanking, consent, and commercialization in international genetics research: The Type 1 Diabetes Genetics Consortium. Clin Trials 2010;7(1_Suppl):S33-S45.

77. Mitchell PB, Ziniel SI, Savage SK, et al. Enhancing Autonomy in Biobank Decisions: Too Much of a Good Thing? J Empir Res Hum Res Ethics 2018;13:125-138.

78. Harris J, Graue M, Dunning T, et al. Involving people with diabetes and the wider community in diabetes research: A realist review protocol. Syst Rev 2015;4:146.

79. Kamuya DM, Marsh V, Kombe FK, Geissler PW, Molyneux SC. Engaging communities to strengthen research ethics in low-income settings: Selection and perceptions of members of a network of representatives in coastal Kenya. Dev World Bioeth 2013;13:10-20.

80. Nyika A, Chilengi R, Ishengoma D, Mtenga S, Thera MA, Sissoko MS. Engaging diverse communities participating in clinical trials: Case examples from across Africa. Malar J 2010;9:86.

81. Adonis T. Patient-Driven Research Agenda Setting in the Netherlands [Qualitative Study]. Amsterdam: Vrije Universiteit Amsterdam; 2016.

82. Sciences SFoMH. Biobanking and Beyond Stellenbosch Faculty of Medicine \& Health Sciences: Youtube; 2016 [cited 201813 July 2018]. Funded by the National Institute of Health, the Centre for Medical Ethics and Law at Stellenbosch University, South Africa produced this educational video "Biobanking and Beyond." The purpose of this video is to inform patients and the general population about the process of taking part in research, and the role of biobanks in medical research. This video takes you on a patient's journey: from meeting the medical doctor, discussing concerns about taking part in research and donating samples, to finding out about the biobank and its role and activities.]. Available from: https://youtu.be/QjSfIA6_bfc.

83. Wazny K, Chan KY. Identifying potential uses of crowdsourcing in global health, conflict, and humanitarian settings: An adapted CHNRI (Child Health and Nutrition Initiative) exercise. J Global Health 2018;8:020704.

84. Conrad EJ, Becker M, Powell B, Hall KC. Improving Health Promotion Through the Integration of Technology, Crowdsourcing, and Social Media. Health Promot Pract 2018:1524839918811152.

85. Strauss RP, Sengupta S, Quinn SC, Goeppinger J, Spaulding C, Kegeles SM. The role of community advisory boards: Involving communities in the informed consent process. Am J Public Health 2001;91:1938-1943.

86. Marlett N, Shklarov S, Marshall D, Santana MJ, Wasylak T. Building new roles and relationships in research: A model of patient engagement research. Qual Life Res 2015; 24:1057-1067.

87. Oral abstracts of the 22nd International AIDS Conference, 23-27 July 2018, Amsterdam, the Netherlands. J Int AIDS Soc 2018;21(S6):e25148.

88. Szilagyi PG, Shone LP, Dozier AM, Newton GL, Green T, Bennett NM. Evaluating community engagement in an academic medical center. Acad Med 2014;89:585-595.

89. Staniszewska S, Brett J, Mockford C, Barber R. The GRIPP checklist: Strengthening the quality of patient and public involvement reporting in research. Int $\mathbf{J}$ Technol Assess Health Care 2011;27:391-399.

90. Khodyakov D, Stockdale S, Jones A, Mango J, Jones F, Lizaola E. On measuring community participation in research. Health Educ Behav 2012;40:346-354.

91. Jagosh J, Pluye P, Macaulay AC, et al. Assessing the outcomes of participatory research: Protocol for identifying, selecting, appraising and synthesizing the literature for realist review. Implement Sci 2011;6:24.

92. Pinsoneault LT, Connors ER, Jacobs EA, Broeckling J. Go slow to go fast: Successful engagement strategies for patient-centered, multi-site research, involving academic and community-based organizations. J Gener Intern Med 2018;34:125-131.

93. Mullins CD, Abdulhalim AM, Lavallee DC. Continuous patient engagement in comparative effectiveness research. JAMA 2012;307:1587-1588.

Address correspondence to: Keymanthri Moodley, MBChB, MFamMed, MPhil, FCFP (SA), Executive MBA, DPhil Department of Medicine Faculty of Medicine and Health Sciences Centre for Medical Ethics \& Law Stellenbosch University P.O. Box 241

Cape Town 7505 South Africa

E-mail:km@sun.ac.za 